\title{
Measuring surgical outcomes in neurosurgery: implementation, analysis, and auditing a prospective series of more than 5000 procedures
}

\author{
Clinical article
}

\author{
Philip V. Theodosopoulos, M.D., Andrew J. Ringer, M.D., \\ Christopher M. McPherson, M.D., Ronald E. Warnick, M.D., Charles Kuntz IV, M.D., \\ Mario Zuccarello, M.D., and John M. Tew JR., M.D. \\ Department of Neurosurgery, University of Cincinnati Neuroscience Institute and University of Cincinnati \\ College of Medicine; and Mayfield Clinic, Cincinnati, Ohio
}

Object. Health care reform debate includes discussions regarding outcomes of surgical interventions. Yet quality of medical care, when judged as a health outcome, is difficult to define because of impediments affecting accuracy in data collection, analysis, and reporting. In this prospective study, the authors report the outcomes for neurosurgical treatment based on point-of-care interactions recorded in the electronic medical record (EMR).

Methods. The authors' neurosurgery practice collected outcome data for 19 physicians and ancillary personnel using the EMR. Data were analyzed for 5361 consecutive surgical cases, either elective or emergency procedures, performed during 2009 at multiple hospitals, offices, and an ambulatory spine surgery center. Main outcomes included complications, length of stay (LOS), and discharge disposition for all patients and for certain frequently performed procedures. Physicians, nurses, and other medical staff used validated scales to record the hospital LOS, complications, disposition at discharge, and return to work.

Results. Of the 5361 surgical procedures performed, two-thirds were spinal procedures and one-third were cranial procedures. Organization-wide compliance with reporting rates of major complications improved throughout the year, from $80.7 \%$ in the first quarter to $90.3 \%$ in the fourth quarter. Auditing showed that rates of unreported complications decreased from $11 \%$ in the first quarter to $4 \%$ in the fourth quarter. Complication data were available for 4593 procedures $(85.7 \%)$; of these, no complications were reported in 4367 (95.1\%). Discharge dispositions reported were home in $86.2 \%$, rehabilitation center in $8.9 \%$, and nursing home in $2.5 \%$. Major complications included culture-proven infection in $0.61 \%, \mathrm{CSF}$ leak in $0.89 \%$, reoperation within the same hospitalization in $0.38 \%$, and new neurological deficits in $0.77 \%$. For the commonly performed procedures, the median hospital LOS was 3 days for craniotomy for aneurysm or intraaxial tumor and less than 1 day for angiogram, anterior cervical discectomy with fusion, or lumbar discectomy.

Conclusions. With prospectively collected outcome data for more than 5000 surgeries, the authors achieved their primary end point of institution-wide compliance and data accuracy. Components of this process included staged implementation with physician pilot studies and oversight, nurse participation, point-of-service data capture, EMR form modification, data auditing, and confidential surgeon reports.

(http://thejns.org/doi/abs/10.3171/2012.7.JNS111622)

\section{KeY Words • outcome • outcome assessment • complication rate}

$\mathrm{W}$ ITH the significant amount of health care expenditures related to surgical procedures and this climate of health care reform, our focus on outcomes of surgical interventions appears timely and important. The CMS initiated a process to evaluate hospital performance for measures of outcome of care (www. hospitalcompare.hhs.gov). As a result, SCIPs were implemented, mostly focusing on compliance with periopera-

Abbreviations used in this paper: $\mathrm{ACDF}=$ anterior discectomy and fusion; CMS = Centers for Medicare and Medicaid Services; $\mathrm{EMR}=$ electronic medical record; LOS = length of stay; SCIP = Surgical Care Improvement Project; VA = Veterans Administration. tive process measures shown to positively impact patient outcome.

Although the health care reform debate has included discussions regarding surgical outcomes, quality of medical care, judged as an outcome, is difficult to define. Many impediments arise with outcome collection, measurement, and reporting. In most medical fields, including neurosurgery, the literature is replete with prospective studies reporting outcomes of specific disease entities and treatment interventions..$^{1,10,11,18-20}$ However, even accurate data may be limited outside the realm of clinical trials in their applicability to clinical practice. Secondary data set analyses, many with good statistical power and large pop- 
P. V. Theodosopoulos et al.

ulations, face the constraints of being retrospective analyses of de-identified data, limited by coding inaccuracies of hospital billing information. Prospective multiinstitutional, disease-specific registries have been reported, but their usefulness is constrained by the intrinsic variability in data accuracy as reported by the various contributors and the lack of effective auditing. ${ }^{2,5,16}$ Increasing evidence indicates that compliance with process measures, such as SCIPs implemented by the CMS, is limited in its translation into quality outcomes for patients undergoing surgery. ${ }^{12}$

In the process of improving the quality of health care, accurate measurement of health outcomes emerges as the first necessary implementation step. Development of a quality of care assessment that is comprehensive, accurate, and auditable can be achieved by the prospective study of individual patient outcomes, collected at various points of service after surgical intervention, along clinically relevant validated measures recorded in an EMR. We undertook this project as a feasibility study of accurate surgical outcome collection in neurosurgery. Our hypothesis is that accurate surgical outcome information can be successfully and accurately collected in the setting of a large neurosurgical practice. In this prospective study, we report on our primary end points of institutionwide compliance with the process and accuracy of recorded information. Surgical outcomes as measured by procedure- and disease-specific measures were secondary end points and are only reported in a limited way, that is, as a general accuracy guideline for our results compared with the plethora of outcomes data already published on the various procedures. Our discussion follows with the lessons learned in the design, implementation, and auditing of our system; we hope that our experience will help refine outcome collection processes that can be applied, not only to other neurosurgery practices, but also to other medical specialty practices. The ultimate goal of this process is overall improvement of neurosurgical care mitigated by the focus placed on outcome collection. We are currently in the process of analyzing the longer-term patient care quality effect of the outcomes initiative and will be reporting these data in a later publication.

\section{Methods}

In an institution-wide initiative, the Mayfield Clinic, a single-specialty academic neurosurgery group, prospectively began to collect surgical case outcome data in 2006. Data were collected by physicians and ancillary personnel using the EMR system (Centricity, General Electric). Our practice includes 19 neurosurgeons who operate in multiple hospitals, including the University of Cincinnati Hospital (the only Level I trauma center in the region) and our ambulatory spine surgery center. Data were compiled and analyzed for 5361 consecutive elective and emergency surgical cases performed during 2009 to represent our often performed procedures $(>60 /$ year) (Table 1). The insurance payer information for all patients is listed in Table 2.

The Outcomes Project was overseen by a task force of physicians, nurses, and ancillary personnel and was led
TABLE 1: Commonly performed procedures performed during 2009

\begin{tabular}{lc}
\hline \multicolumn{1}{c}{ Procedure Performed } & $\begin{array}{c}\text { No. of } \\
\text { Patients }\end{array}$ \\
\hline craniotomy for aneurysm & 98 \\
diagnostic angiogram & 342 \\
ACDF & 734 \\
craniotomy for hematoma, nontraumatic & 117 \\
craniotomy for hematoma, traumatic subdural & 62 \\
craniotomy for intraaxial tumor & 126 \\
stereotactic radiosurgery for intraaxial tumor & 163 \\
kyphoplasty & 103 \\
lumbar discectomy/decompression, no instrumentation & 1184 \\
lumbar discectomy/decompression, w/ instrumentation & 514 \\
transsphenoidal resection of sellar/parasellar mass & 67 \\
spinal cord stimulation & 198 \\
deep brain stimulation for movement disorder & 86 \\
ventriculoperitoneal shunt, initial or revision & 109 \\
\hline
\end{tabular}

by a surgeon and a clinical nurse; this nurse's full-time job was dedicated to project implementation, data analysis, and auditing of the results. Based on the initial data parameters specified by the task force, we redesigned our EMR forms. During the initial 1-year trial period (20072008), 3 surgeons voluntarily monitored and troubleshot problems that were encountered using the process in their practices. Thereafter, the data collection process was integrated throughout our organization.

Outcome data were recorded on the health and functional status of the patient at specified point-of-service encounters in the immediate and delayed postoperative setting. The data format was tailored according to the preferences of the physician practices: those using dictations completed designated outcomes forms, whereas physicians using prespecified EMR forms filled in specific outcome fields. Data were recorded by physicians, nurses, nurse practitioners, medical secretaries, and medical assistants. If a nonphysician team member completed the outcome form, it was routed to the responsible physician for electronic sign-off. Data included LOS, major and minor complications, disposition at hospital discharge, return to work, scores on relevant validated scales (Oswestry Disability Index for patients with spinal disease, the Karnofsky Performance Scale and Eastern Cooperative Oncology Group Performance Status for patients with tumors, and the modified Rankin Scale for patients with vascular conditions and trauma), and symptom severity.

Complications were classified according to a combined scheme of type and severity along the lines of the National Cancer Institute Common Terminology Criteria for Adverse Effects (CTCAE version 4.0, ctep.cancer. gov). Major complications were defined as Grade 3 or 4 adverse effects along the system organ classes of nervous system disorders, infections and infestations, and surgical and medical procedures as deemed appropriate, or as Grades 2 through 4 for the development of a new stroke or new seizure disorder. Minor complications were defined 
TABLE 2: The insurance-payer distribution for all patients

\begin{tabular}{lc}
\hline Insurance Payer & \% Patients \\
\hline Blue Shield & 20.1 \\
commercial & 26.9 \\
Medicaid & 8.6 \\
Medicare & 34.6 \\
self-pay & 6.1 \\
workers' compensation & 3.2 \\
other & 0.5 \\
\hline
\end{tabular}

as Grade 1 or 2 adverse effects or medical complications that, although related to the perioperative state of the patient, were not thought to be a direct result of the surgical intervention. Both minor and major complication EMR fields included a "none" rating to differentiate between the lack of complication or incomplete field (Appendix).

On an ongoing basis, compliance in recording outcome data was assessed. The results tabulated electronically every quarter included compliance (that is, rates of EMR outcome form completion), rates of major complications, specific clinical outcomes per procedure, LOS, and surgeon-specific clinical outcomes. We also compiled procedure-specific outcome data for 5 of our most commonly performed spinal and cranial procedures: craniotomy for aneurysm or intraaxial tumor, diagnostic cerebral angiogram, $\mathrm{ACDF}$, and lumbar discectomy without instrumentation.

\section{Auditing and Reporting Outcome Data}

Our auditing process included a quarterly data review of randomly chosen (computer-generated) patient charts (5\% for each surgeon). The audit included all information on the EMR and additional information from the hospital data systems. The great majority of the charts audited had the outcomes fields already completed by the physician teams. Our auditing process did not focus on charts with incomplete record fields. For all outcome categories identified, inconsistencies were reviewed and, if found discrepant, were adjudicated by the physician manager. The process by which the physician manager resolved an inconsistency was straightforward with the inclusion of all data reviewed and, when questionable, weighed toward overreporting a complication. For example, a lumbar laminectomy procedure had an outcome form that reported LOS information, discharge disposition, and no major complications; however, according to the hospital chart, a wound swab was positive for Staphylococcus aureus growth in culture. Therefore, the record was revised to include culture-proven infection (major complication), and the inconsistency was noted as a missed complication.

Quarterly reports included misreported complication rates and a confidential outcome review for each surgeon's most-often performed procedures. Results of clinical outcomes by procedure were quantified, both for each surgeon and the clinic as a whole, quarterly and for the duration of a surgeon's career at Mayfield Clinic. Blinded data were reviewed by our Risk Management Committee; this review was designed as an assessment of the organization's standards of quality rather than as a comparison among surgeons. If performance exceeds 2 standard deviations below the clinic norm, data are unblinded for assessment and intervention.

\section{Results}

During the 1-year period, of the 5361 surgical procedures performed by 19 neurosurgeons, about two-thirds were spinal procedures and slightly more than one-third were cranial procedures. Considering the complication data available for 4593 procedures (85.7\%), 4367 (95.1\%) had no complications. The rates of major complications reported included culture-proven infection (0.61\%), CSF leakage $(0.89 \%)$, reoperation within the same hospitalization $(0.38 \%)$, and new neurological deficits $(0.77 \%$; Fig. 1$)$. Discharge disposition included home in $86.2 \%$ of cases, rehabilitation center in $8.9 \%$, and nursing home in $2.5 \%$; $0.76 \%$ of patients died.

\section{Compliance}

Our first primary end point of organization-wide compliance (rates for completed fields in the EMR) improved for each successive quarter. The EMR field data were complete in $80.7 \%$ of records for major complications and $48.9 \%$ for discharge disposition during the first quarter; these rates increased to $90.3 \%$ and $58.9 \%$, respectively, during the fourth quarter. Along with review of quarterly data reports, the physicians and staff received training in process implementation and reporting accuracy. We evaluated the rates of completion for the major complication field (that is, its absence or presence). For instance, the field for major complications for ACDF was complete for $79 \%$ in the first quarter and $95 \%$ in the fourth quarter following clinic-wide education about outcome reporting. Additionally, interventions targeted to improve reporting proved to be effective. For the physician office reporting on the presence or absence of major complications associated with diagnostic angiography, field completion was $41 \%$ in the first quarter and $74 \%$ in the fourth quarter. In comparison, completion rates for basic required universal fields, that is, patient weight and height, were $80.8 \%$ and $79.5 \%$, respectively.

\section{Auditing}

Our second primary end point of accuracy of information recorded improved with each successive quarter. Of 5361 procedures performed, 265 charts $(4.9 \%)$ were audited. During the study period, we identified missing data fields for major complications in $8.5 \%$ of the charts reviewed; however, this rate steadily declined for each successive quarter, starting at $11 \%$ and ending with $4 \%$. Auditing was conducted 3 months after the quarter's end to ensure adequate time for complication recording.

\section{Procedure-Specific Outcomes}

Major complications by procedure are shown for 5 commonly performed procedures (craniotomy for aneurysm or intraaxial tumor, diagnostic cerebral angiogram, 




FIG. 1. Prospective study to identify quarterly $(Q)$ rates of major complications for 5361 procedures (two-thirds spinal and one-third cranial) performed by 19 neurosurgeons during 2009 (09). Complication data from the EMR were available for 4593 procedures (85.7\%); of these, no complications were recorded in $95.1 \%$.

ACDF, and lumbar discectomy) (Fig. 2). The median hospital LOS was 3 days for craniotomy for aneurysm or intraaxial tumor and less than 1 day for angiography, $\mathrm{ACDF}$, or lumbar discectomy (Fig. 3).

\section{Reporting}

Surgeon outcomes were compiled quarterly for each surgeon's most commonly performed procedures. Considering that the acuity and complexity of the disease naturally varied for each patient, no weighing process adjusted for these differences. However, each surgeon received his or her own quarterly data, his or her lifetime data with the clinic, and a glimpse into significant deviations from the clinic average. Our risk management committee reviewed individual surgeon reports in a blinded fashion to identify possible outlying results. Throughout this process, we found no instance that required unblinding and further review of the data.

\section{Discussion}

Evaluating our prospectively collected outcome data for more than 5000 surgeries during 2009, we achieved our primary end point of institution-wide compliance and data accuracy as evidenced by the improvement in reporting compliance for each successive quarter and the decreased rate of missed complications reported. Our compliance rates, although not yet optimal, continue to progress as we aim toward completely capturing all outcome data. As indicated in Results, completion of even rudimentary information fields (for example, height and weight) required for all patients hovered around $80 \%$ during the same period; this rate indicates a relatively high familiarity with the EMR but by no means a universal capture of all pertinent patient information. During the several years of development and pilot studies, we refined the components of this process to include staged implementation with physician oversight, nurse participation, point-of-service data capture, EMR form modification, targeted interventions in underperforming physician's offices, data auditing, and confidential reports to the surgeon.

With no universally accepted definition for quality of care, several outcomes studies have used surrogates for quality of care including patient-centered data, such



FIG. 2. Rates of the major complications associated with 5 commonly performed procedures (> 60/year) reported quarterly in 2009. 


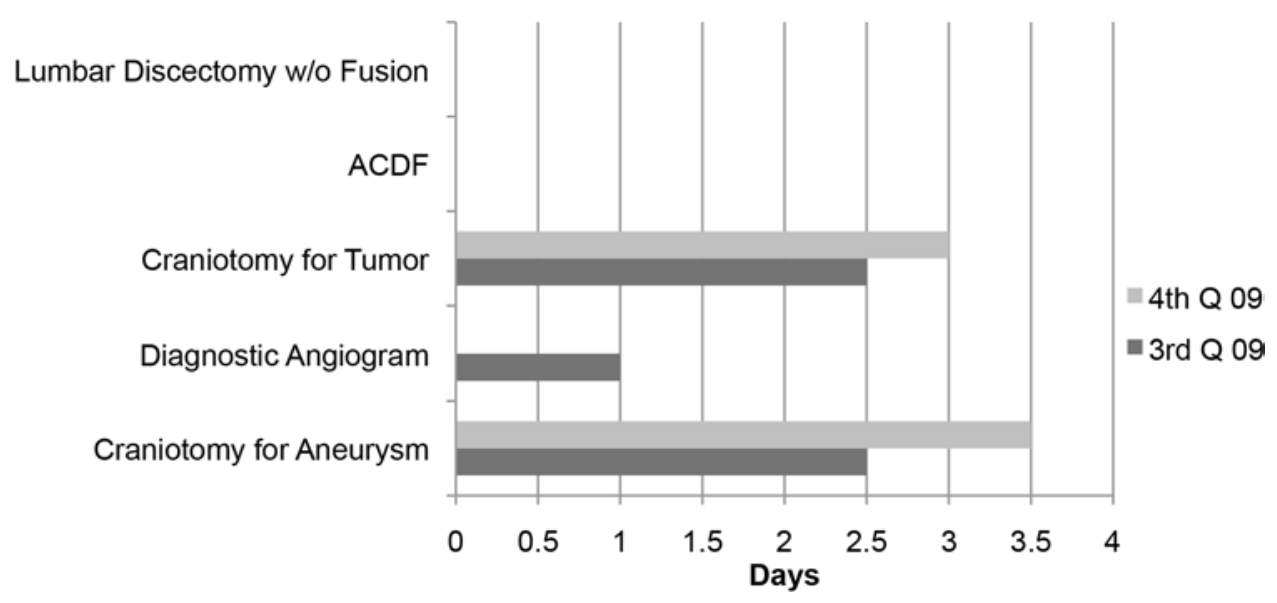

FIG. 3. Hospital LOS for 5 commonly performed procedures. Because lumbar laminectomy without fusion and ACDF are now mostly performed as outpatient surgery in our practice, there is no LOS. In auditing our data for this outcome, we noted that this EMR field was incomplete for Quarters 1 and 2. Troubleshooting this process, we then found that our billing department could readily provide records for hospital LOS (Quarters 3 and 4).

as rates of mortality, major complications, and discharge disposition to home, and systemic indicators, such as hospital-level diagnosis-based data, ${ }^{9}$ malpractice claims, ${ }^{13}$ and accepted process adherence data. ${ }^{17}$ These surrogates, although not accurately reflecting health care outcomes for individual patients, are nonetheless powerful indicators of certain dimensions of care averaged over many patients with similar conditions or similar treatments within specific-care settings. Analysis of such secondary databases is often accurate and relatively straightforward. As such, early outcomes research was performed in this fashion.

The study of secondary databases to derive quality of health outcomes has limitations related to data validity and general applicability of conclusions. A primary limitation is the inability to correlate data from individual patient hospitalizations. Because most secondary database information is de-identified, analysis of comorbidity and disease severity is limited. Information on secondary hospital-billing ICD-9 codes, traditionally used to stratify disease severity, can often be inaccurate and incomplete. Most importantly, the nature of secondary data makes auditing of the accuracy of individual patient data recorded impossible, disallowing data entry error recognition and correction.

Increasing evidence shows that secondary data analyses and surrogates of direct patient level outcomes, particularly for surgical interventions, may not accurately represent quality of care. In a 2010 study, Nicholas et al. ${ }^{12}$ found low correlation between rates of compliance with perioperative process of care as suggested by the CMS SCIPs and postoperative patient outcomes in 2000 US hospitals. The authors emphasized a shift toward either more robust process measures or away from process measures altogether.

Collecting primary outcome data has limitations as well. The data can be difficult to measure accurately, or they can be easy to measure for small subgroups but difficult to generalize for other applications. One example is the measurement of patient mortality after surgical inter- vention, such as the system used to track mortality rates after coronary artery bypass graft surgery for hospitals and individual surgeons in New York state. ${ }^{7}$ This initiative was widely commended and resulted in improvements in health care utilization and cardiac surgery outcomes. Mortality, although straightforward to measure, poses significant limitations when applied as a primary outcome measure, even related to inpatient surgical care. As Dimick et al. ${ }^{4}$ suggested regarding surgical patients, a procedure must have a significantly high mortality rate if the rate is to function as a quality indicator. Similar to most noncardiac surgeries, the majority of neurosurgical procedures have nearly negligible mortality rates. Our overall mortality rate is $0.76 \%$ and $0.46 \%$ when adjusted to exclude emergency operative interventions for intracerebral nonaneurysmal hemorrhage; this rate is in the range of previously reported studies for neurosurgery $(0.13 \%-1.3 \%)^{5,14}$ but is certainly much lower than the $3.3 \%$ $\pm 0.6 \%$ rate for inpatient admissions for coronary artery bypass graft (AHRQ, http://www.ahrq.gov/qual/nhqr04/ fullreport/Data_Tables/DT1_50.htm).

The alternative to primary outcome measures, such as surgical mortality, that are simple to define and collect but difficult to generalize may be a range of measures that can be difficult to define and measure but more relevant to health care delivery in most settings. In a VA study of perioperative outcomes, Khuri et al. ${ }^{8}$ prospectively collected data from 400,000 surgical procedures performed at 123 VA hospitals. Data collected included rates of mortality and major complications, which are generally accepted as relevant and easily generalizable primary outcome measures. Patients reported 30-day outcomes and complications to the study coordinators in a process that allowed for correlation between the data and each patient's chart information. Although the process was effective, accuracy of data collection and auditing was limited; outcomes were reported in part by patients themselves and, given the large size of the study, only samples of patients were recorded and audited. Yet, similar to our experience, the authors found that the presence of on-site 
trained clinical nurse coordinators ensured accurate collection, validity, and reliability for the data.

In a follow-up VA study, Ghaferi et al. ${ }^{6}$ reviewed data for all general and vascular surgeries at 180 VA hospitals where expected mortality rates were greater than $1 \%$. The authors created a prospective, de-identified, patient data registry that included mortality and morbidity hospitallevel data. However, again, the rates estimated were based on data collected in a random sampling of patients who underwent each procedure.

In the field of neurosurgery, notable examples of outcome studies based on disease-specific multiinstitutional databases are the Glioma Outcome Project collecting data on craniotomies for primary brain tumors, and the Scoliosis Research Society morbidity and mortality database for spinal surgery. ${ }^{2,5,16}$ In both registries, data collection was standardized and yielded information for a number of surgical procedures. Yet, as they were multiinstitutional databases, the information was de-identified and could not be audited. Accuracy is ensured only by the completeness at initial input of the information by the primary provider, a weakness that is shared with most secondary database analyses.

Early in our process, we proposed that an effective and accurate system for outcome data collection should be EMR based, focused on point-of-service data collection, and coupled to a strong auditing process. Outcome information was collected within the EMR for various parameters that were important to patients, physicians, and payers along many point-of-care interactions. Auditing confirmed the accuracy of our process in a surgical subspecialty practice.

In agreement with a recently published smaller study on spine surgery that found prospectively collected, ICD9 based complication rates to be more accurate than rates derived via retrospective analysis of the same data, ${ }^{21}$ we found that our prospectively collected complication rates were similar to those of published smaller prospective disease-specific studies that, by design, were significantly more detailed. Our reported rate of major complications after craniotomy for an intraaxial brain tumor was $10.3 \%$, similar to the $13 \%$ rate reported in a large prospective study by Sawaya et al. ${ }^{14}$ Our major complication rates for $\mathrm{ACDF}$ and lumbar discectomy were $1.6 \%$ and $2.4 \%$, respectively, similar to the $2.4 \%$ and $3.6 \%$ overall complication rates for such cases from the Scoliosis Research Society morbidity and mortality database..$^{5,16}$

\section{Study Limitations}

Our process has several limitations, including that our data were typically provided by the primary surgeons. As others have shown, there is an inherent reservation for practitioners when individual providers' outcomes data become public. ${ }^{3}$ However, our auditing process revealed a low rate of reported inaccuracies. We attribute this low reporting bias to the fact that all members of the health care team were empowered to record outcome information at each point of service. The accuracy of the information reported by the different-level providers (physician, nurse, and medical assistant) was not addressed in this study and may be another important source of bias.
Nonetheless, expansion of the auditing review beyond the random 5\% of each surgeon's cases may be prudent. Additionally, targeted review of random samples of the charts with unreported complications may reveal a bias of underreporting of major complications when they occur; however, our auditing process to date has not revealed any differences in accuracy between the charts with complete or incomplete outcome fields.

Despite the adoption of the National Cancer Institute Common Terminology Criteria for Adverse Effects in defining complication severity, what constitutes a procedure-related complication remains subject to debate. Sentinel events or laboratory results are not open to interpretation, yet other outcomes are harder to define. Transient ones, such as the postoperative development of a new neurological deficit, may be expected to improve in the immediate postoperative period. Others, such as fusion following spinal instrumentation, have no universally used diagnostic criteria. ${ }^{15}$ Timing of such events can also diminish reporting accuracy, such as the case of a spinal instrumentation failure identified 6 months postoperatively that was in fact previously unrecognized yet related to the initial procedure.

The definitions of major and minor complications were relative and should not be interpreted as important versus unimportant ones. Although these designations were initially made for practical reasons related to the design complexity of the EMR forms, our experience has shown that redesigning the forms to include all relevant complications (both surgical and medical) under a single field may be better.

Detailed analysis of disease-specific outcomes rather than procedure-related outcomes may allow the identification of inaccuracies that are not yet obvious. Rates of compliance with established guidelines for certain diseases were not tabulated and will be reported in subsequent studies. Reporting accuracy for comorbidities varied, given that, at times, there was a lack of available clinical history for the patient, particularly in cases of an urgent or emergency procedure. This limitation, combined with the frequent multiorgan effects of severe brain and spine injury, made identification of procedure-related complications inadequate at times. Although emergency procedures constitute only a small fraction of the total volume, these cases still may have caused inaccuracies in our reported rates.

Last, but by no means least, the patient population under study is of paramount importance with respect to outcomes of surgical intervention. As the primary end points were related to implementation of our outcome collection process, we purposefully did not significantly delve into patient characteristics. Although representative of any other setting, our practice spans the world of academic and private practice neurosurgery and covers the entire breadth of neurosurgical procedures performed nationally. The payer mix and the ratio of emergency to elective surgery are representative of many other large practices.

Despite these limitations, our large study during this multiyear endeavor has brought many lessons bearing on the development and implementation of a process that can be generalized for many medical settings, thus helping 
practices achieve easier, quicker success in collecting outcome data. First, detailed planning of the parameters to be measured, with good definitions and time points, was crucial in facilitating EMR design and implementation, and avoiding down-the-line modifications that disrupt the process. Second, given that the data gathering involved most of our organization, system-wide education on this process was imperative. Focused interventions in underperforming offices often showed misunderstandings about the process; these were generally well accepted and effective. Third, a trial period, when a few technology-apt physicians input data and identified systemic problems, was important for a smooth organization-wide implementation. Fourth, auditing is a must. Our auditing process surveyed a random percentage of patients' charts, although alternative ways can be equally effective. Finally, quarterly presentations reviewed by all those involved served as a powerful motivator for the continued success of the initiative.

The overarching goal of this process is the improvement of quality of surgical care that we provide our patients. During these initial 5 years of development, we have not yet proven that quality enhancement is a measurable result of our intervention. Qualitatively, however, the culture of our entire organization has benefited from the increased attention to outcomes. That is, clinic-wide our point-of-care interactions are designed to immediately report untoward events directly on specific outcome forms that are now familiar to all health care providers. This improvement has provided a focus on overall patient care that was previously tedious, fragmented, and often inaccurate.

\section{Conclusions}

The future direction of our effort focuses on enhancing the accuracy of outcome information collected. Analyzing disease-specific data, interfacing with the neurosurgical ICU database to improve the accuracy of reporting complications in the face of comorbidities, introducing risk adjustment for disease severity, and broadening the auditing process are all processes underway. As accurate outcome data become available, the biggest challenge will be determining how to use the information creatively in ways that effect changes in treatment and ensure outcome improvement.

\section{Appendix: Complications}

CSF
Major
excessive intraoperative bleeding
postoperative hemorrhage
implant failure
culture-proven infection
myocardial infarction
new-onset diabetes mellitus
reoperation within the same hospitalization
new neurological deficit
pulmonary embolism
pneumonia
new seizure disorder

stroke unplanned reoperation wound dehiscence

\section{Minor}

allergic reaction

deep venous thrombosis

hoarseness

prolonged inpatient stay

swallowing dysfunction

presumed wound infection treated with antibiotics

wound hematoma (not requiring surgical intervention)

\section{Disclosure}

Dr. Kuntz has ownership in Mayfield Clinic, Christ Hospital Spine Surgery Center, Precision Radiotherapy, Priority Consult, and CKIV Alignment. He is also a consultant for Synthes and DePuy. He receives support of non-study related clinical or research effort from AOSpine, Stryker, Synthes, and DePuy.

Author contributions to the study and manuscript preparation include the following. Conception and design: Theodosopoulos, Ringer, McPherson, Warnick, Kuntz, Tew. Acquisition of data: Theodosopoulos, Ringer, McPherson, Warnick, Kuntz. Analysis and interpretation of data: all authors. Drafting the article: all authors. Critically revising the article: all authors. Reviewed submitted version of manuscript: all authors. Statistical analysis: Theodosopoulos, Ringer, McPherson, Warnick, Tew.

\section{References}

1. Buchbinder R, Osborne RH, Ebeling PR, Wark JD, Mitchell $\mathrm{P}$, Wriedt $\mathrm{C}$, et al: A randomized trial of vertebroplasty for painful osteoporotic vertebral fractures. N Engl J Med 361: 557-568, 2009

2. Chang SM, Parney IF, Huang W, Anderson FA Jr, Asher AL, Bernstein M, et al: Patterns of care for adults with newly diagnosed malignant glioma. JAMA 293:557-564, 2005

3. Chassin MR, Hannan EL, DeBuono BA: Benefits and hazards of reporting medical outcomes publicly. N Engl J Med 334: 394-398, 1996

4. Dimick JB, Welch HG, Birkmeyer JD: Surgical mortality as an indicator of hospital quality: the problem with small sample size. JAMA 292:847-851, 2004

5. Fu KM, Smith JS, Polly DW Jr, Perra JH, Sansur CA, Berven $\mathrm{SH}$, et al: Morbidity and mortality in the surgical treatment of 10,329 adults with degenerative lumbar stenosis. Clinical article. J Neurosurg Spine 12:443-446, 2010

6. Ghaferi AA, Birkmeyer JD, Dimick JB: Variation in hospital mortality associated with inpatient surgery. N Engl J Med 361:1368-1375, 2009

7. Hannan EL, Kilburn H Jr, Racz M, Shields E, Chassin MR: Improving the outcomes of coronary artery bypass surgery in New York State. JAMA 271:761-766, 1994

8. Khuri SF, Daley J, Henderson W, Barbour G, Lowry P, Irvin G, et al: The National Veterans Administration Surgical Risk Study: risk adjustment for the comparative assessment of the quality of surgical care. J Am Coll Surg 180:519-531, 1995

9. Longo DR, Land G, Schramm W, Fraas J, Hoskins B, Howell $\mathrm{V}$ : Consumer reports in health care. Do they make a difference in patient care? JAMA 278:1579-1584, 1997

10. Mehta MP, Tsao MN, Whelan TJ, Morris DE, Hayman JA, Flickinger JC, et al: The American Society for Therapeutic Radiology and Oncology (ASTRO) evidence-based review of the role of radiosurgery for brain metastases. Int J Radiat Oncol Biol Phys 63:37-46, 2005

11. Molyneux AJ, Kerr RS, Yu LM, Clarke M, Sneade M, Yarnold JA, et al: International subarachnoid aneurysm trial (ISAT) of neurosurgical clipping versus endovascular coiling in 2143 


\section{P. V. Theodosopoulos et al.}

patients with ruptured intracranial aneurysms: a randomised comparison of effects on survival, dependency, seizures, rebleeding, subgroups, and aneurysm occlusion. Lancet 366: 809-817, 2005

12. Nicholas LH, Osborne NH, Birkmeyer JD, Dimick JB: Hospital process compliance and surgical outcomes in medicare beneficiaries. Arch Surg 145:999-1004, 2010

13. Rolph JE, Kravitz RL, McGuigan K: Malpractice claims data as a quality improvement tool. II. Is targeting effective? JAMA 266:2093-2097, 1991

14. Sawaya R, Hammoud M, Schoppa D, Hess KR, Wu SZ, Shi WM, et al: Neurosurgical outcomes in a modern series of 400 craniotomies for treatment of parenchymal tumors. Neurosurgery 42:1044-1056, 1998

15. Skolasky RL, Maggard AM, Hilibrand AS, Northrup BE, Ullrich CG, Albert TJ, et al: Agreement between surgeons and an independent panel with respect to surgical site fusion after single-level anterior cervical spine surgery: a prospective, multicenter study. Spine (Phila Pa 1976) 31:E503-E506, 2006

16. Smith JS, Fu KM, Polly DW Jr, Sansur CA, Berven SH, Broadstone PA, et al: Complication rates of three common spine procedures and rates of thromboembolism following spine surgery based on 108,419 procedures: a report from the Scoliosis Research Society Morbidity and Mortality Committee. Spine (Phila Pa 1976) 35:2140-2149, 2010

17. Stevenson KS, Gibson SC, MacDonald D, Hole DJ, Rogers $\mathrm{PN}$, Byrne DS, et al: Measurement of process as quality con- trol in the management of acute surgical emergencies. Br $\mathbf{J}$ Surg 94:376-381, 2007

18. Tsao MN, Mehta MP, Whelan TJ, Morris DE, Hayman JA, Flickinger JC, et al: The American Society for Therapeutic Radiology and Oncology (ASTRO) evidence-based review of the role of radiosurgery for malignant glioma. Int J Radiat Oncol Biol Phys 63:47-55, 2005

19. Turner JA, Ersek M, Herron L, Haselkorn J, Kent D, Ciol MA, et al: Patient outcomes after lumbar spinal fusions. JAMA 268:907-911, 1992

20. Wiebe S, Blume WT, Girvin JP, Eliasziw M: A randomized, controlled trial of surgery for temporal-lobe epilepsy. N Engl J Med 345:311-318, 2001

21. Yadla S, Malone J, Campbell PG, Maltenfort MG, Harrop JS, Sharan AD, et al: Early complications in spine surgery and relation to preoperative diagnosis: a single-center prospective study. Clinical article. J Neurosurg Spine 13:360-366, 2010

Manuscript submitted September 23, 2011.

Accepted July 11, 2012.

Please include this information when citing this paper: published online August 10, 2012; DOI: 10.3171/2012.7.JNS111622.

Address correspondence to: Philip Theodosopoulos, M.D., c/o Editorial Office, Department of Neurosurgery, University of Cincinnati College of Medicine, ML 0515, Cincinnati, Ohio 45267-0515. email: editor@mayfieldclinic.com. 